\title{
Variables y dimensiones aplicadas a la percepción en la calidad del servicio de cajeros automáticos
}

\section{Variables and dimensions applied to the perception in the quality of ATM service}

\author{
Msc. Jacqueline del Pilar Hinostroza Morocho ${ }^{1}$ \\ jhinostroza@gmail.com \\ ORCID: 0000-0002-0479-4528 \\ $\mathrm{PhD}$. José Enrique Townsend Valencia ${ }^{2}$ \\ Jose.townsend@uteg.edu.ec \\ ORCID: 0000-0001-5319-4425
}

Recibido: 1/07/2020; Aceptado: 1/09/2020

\begin{abstract}
RESUMEN
El uso de cajeros automáticos es cada vez más frecuente y exige por parte de los clientes un servicio de calidad. Es un elemento primordial para la bancarización y diversificación de productos financieros, aumentando la gestión por el ofrecimiento de sus servicios 24 horas 7 días a la semana. El avance tecnológico de estos equipos ha conllevado al reemplazo de los cajeros humanos por equipos sin necesidad de intervención humana. La percepción del servicio se encuentra limitada por la no existencia de la valoración de la experiencia recibida del cliente antes, durante y post de su transacción. Se ha considerado difícil medir una percepción del servicio debido a que no existe un representante de la banca para entender y atender los reclamos de su experiencia recibida y en la medida que el cliente queda satisfecho lo que se convierte en una interrogante importante para las entidades bancarias. Este artículo analiza la problemática desde la percepción del cliente en el servicio del cajero automático planteando si la banca comercial puede mantenerse competitiva sin la necesidad de un análisis de percepción, siendo objetivo principal identificar sus variables y dimensiones en la medición del servicio a partir de las aportaciones de varios modelos desde la perspectiva tecnológica. La investigación realizada es de diseño cuantitativo, no experimental, transeccional, con una epistemología positivista. Su alcance es descriptivo para detallar las propiedades y características de cada variable investigada y correlacional para conocer el grado de asociación de cada variable sobre el objeto de estudio con la aplicación de técnicas estadísticas, documentales y de campo.
\end{abstract}

\footnotetext{
${ }^{1}$ Docentes de la Universidad Tecnológica Empresarial de Guayaquil, Ecuador.

2 Docentes de la Universidad Tecnológica Empresarial de Guayaquil, Ecuador.
} 


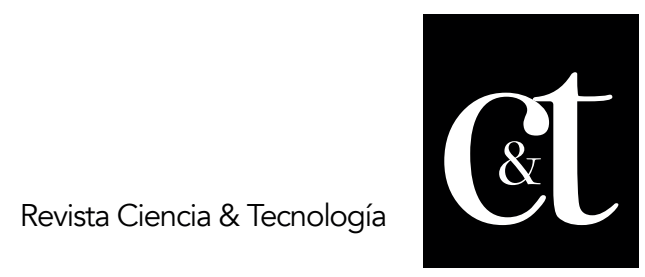

No. 28,31 de octubre de 2020

ISSN impreso: 1390 - 6321

ISSN online: 2661 - 6734

Palabras clave: calidad de servicio, percepción, modelo, cajero automático

\begin{abstract}
The use of ATMs is increasingly frequent in financial institutions and requires quality service from their customers. Key elements are diversification and continuous service during twenty-four hours during the seven days of the week. Technological advancement has led to the replacement of human cashiers, allowing customers to be served without human intervention, withdraw their money, pay for a service or make a deposit. This could be so simple or difficult depending on the type of customer and the complexity of the design of financial institution transactions. Perception of the service is limited due to non-existence of an evaluation regarding the experience received from the client before, during and after the transaction. There is no representative of the bank to understand and address the claims of their experience and to the extent that the client is satisfied which becomes an important question for banks. This article analyzes the problem from the perception of the customer in the service of the ATM proposing if the commercial banking can remain competitive without the need of a perception analysis the main objective being to identify its variables and dimensions in the measurement of the service from the contributions of several models from the technological perspective. The research is a quantitative design, not experimental, transectional with a positivist epistemology, its scope is descriptive to detail the properties and characteristics of each variable investigated and correlational to know the degree of association of each variable on the object of study with the application of statistical, documentary and field techniques.
\end{abstract}

Keywords: quality of service, perception, model, ATM

\title{
Introducción
}

La banca comercial enfocada en el crecimiento y posicionamiento del mercado desarrollan productos de canales electrónicos con el fin de retener y captar nuevos clientes, este crecimiento viene acompañado de equipos y/o programas computacionales que brindan a los clientes la facilidad de poder atenderse sin necesidad de asistir a una oficina bancaria, tal es el caso como la ampliación de las coberturas de los cajeros automáticos o también denominados ATM $^{3}$ que son utilizados como diferenciador por su diversidad de funcionalidades como dispensadores y recicladores.

La característica principal de un cajero automático es el autoservicio de la inmaterialidad de sus productos electrónicos que no requiere la presencia de un representante en cada lugar que ofrece el servicio y que obliga a la banca comercial a establecer procesos y controles al servicio percibido identificando proactivamente eventos erróneos y anómalos presentados en la transacción electrónica que el cliente no informa afectando y que van afectando paulatinamente la confianza y seguridad de la institución bancaria.

\footnotetext{
${ }^{3}$ ATM: Siglas de cajeros automáticos en el idioma inglés, AUTOMATIC TRANSFER MACHINE.
} 


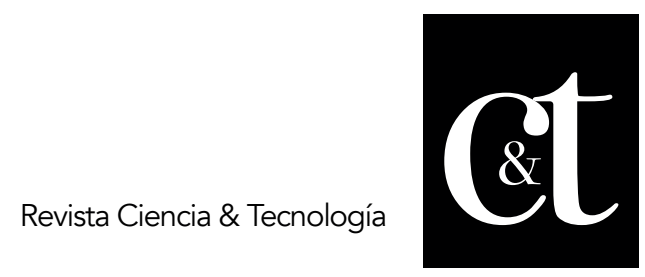

No. 28,31 de octubre de 2020

ISSN impreso: 1390 - 6321

ISSN online: 2661 - 6734

Encontrar las variables que se miden desde la perspectiva del autoservicio identificando sus dimensiones tecnológicas con las aportaciones de modelos especializados en la medición de cajeros automáticos para identificar a partir de sus resultados bajo qué condiciones se puede tomar decisiones operacionales y tecnológicas.

Del Molino (2010) considera la calidad como un conjunto de características que cumplen con los requisitos donde juega un papel importante el cliente definiendo claramente las características que le impacta. Noriaki Kano (1984), define la evaluación de la calidad como la prestación de servicios realizados por una empresa en base a requerimientos, estos requerimientos se los clasifica en esperados que se los entiende como un estándar de la calidad e inesperados que al ser interpretados correctamente pueden dar un valor agregado a la satisfacción del consumidor.

Grönroos (1984) fue uno de los primeros investigadores en proponer una evaluación de la calidad del servicio considerándola como "una actividad o serie de actividades de naturaleza más o menos intangible que no necesariamente tiene lugar a través de interacciones entre el cliente y los empleados de la empresa de servicios", esto es que la calidad percibida está en función del servicio percibido de la empresa que no requiere la asistencia de un representante (Ribeiro, Machado \& Tinoco, 2010).

Grande (2005) define a estos servicios como los intercambios de valores con la finalidad de entregar un resultado deseado a los clientes. El resultado deseado debe medirse, por lo que normalmente se utiliza modelos referenciales de calidad que permiten valorar el proceso; identificando las variables y dimensiones adecuadas que miden la calidad del servicio con el fin de tener un conocimiento real sobre lo que reciben los clientes y presentar de forma estandarizada sus resultados (Berdugo, Barbosa \& Prada, 2016).

En su definición los modelos de calidad se basan en medir las expectativas y las percepciones del servicio. La primera tendencia se basa en el paradigma de la desconfirmación de Oliver (1980) debido a que el consumidor después de utilizar el servicio realiza una discrepancia entre la percepción de los resultados obtenidos y las expectativas creadas previamente antes del servicio, ubicando esta tendencia al modelo Nórdico de Grönroos (1984) y al modelo norteamericano "Service Quality" de Parasuraman, Zeithaml y Berry (1988) denominado SERVQUAL. La segunda tendencia mide solo las percepciones del cliente fundamentando que incluir las expectativas es innecesario e ineficiente en los resultados del servicio.

Entre el modelo que se destaca es Cronin \& Taylor (1992) desarrollado como escala alternativa al modelo SERVQUAL con la diferencia que se concentra en la valoración del desempeño denominado "Service Perfomance" o escala de SERVPERF y elimina la parte que referencia las expectativas de los clientes. El modelo se basa en una serie de preguntas que contrastan la calidad realizada entre las expectativas y las percepciones y la realizada con las percepciones o actitudes de los mismo, analizando más de cerca las implicaciones sobre las actitudes y satisfacción.

\section{Desarrollo}

Varios estudios en el gráfico No 1 resaltan la relación de la percepción de la calidad con los servicios bancarios y sus medios tecnológicos facilitando la conexión de los 


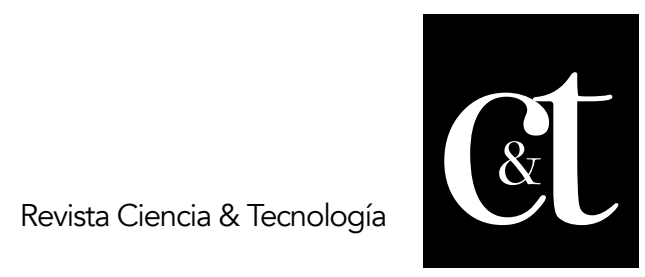

No. 28,31 de octubre de 2020

ISSN impreso: 1390 - 6321

ISSN online: 2661 - 6734

servicios con el comportamiento, la precisión de las transacciones bancarias y la percepción del servicio del hardware y software.

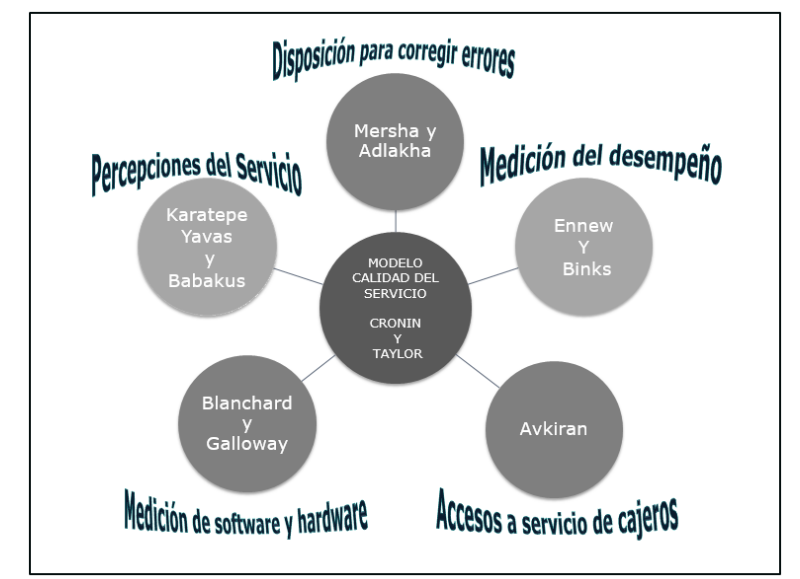

\section{Gráfico 1. Estudios de referencia bancaria}

Fuente: autores

Mersha \& Adlakha (1992) identificaron y clasificaron los atributos de la calidad desde la perspectiva de los clientes ampliando las dimensiones de SERVQUAL en base al modelo de Haywood-Farmer, qué resaltó el conocimiento del servicio oportuno y rápido, su personalización e información. Se clasificó los atributos en buena calidad que resultó ser disposición a corregir los errores, precisión del servicio, confiabilidad y conocimiento; y las características más importantes de la mala calidad en la banca fue el servicio descuidado, la falta de conocimiento del servicio, renuencia a corregir los errores e indiferencia de los empleados (Sangeetha y Mahalingam, 2011).

Avkiran (1994) desarrolló un instrumento multidimensional para medir la percepción en el acceso a los servicios, conducta personal, credibilidad y comunicación de cajeros utilizando como modelo de partida SERVQUAL, pero no pudo reproducir las cinco dimensiones debido a que no se pudo desarrollar una calidad del servicio a medida porque cada servicio se desarrolló en un entorno cultural (Karatepe, Yavas \& Babacus, 2005).

Ennew y Binks (1996) examinaron los problemas relacionados de las expectativas, percepciones y satisfacción en la medición de desempeño de la banca en tres tipos de variables relevantes, la primera consiste en las características específicas del producto, la segunda al producto como proceso y la tercera se centra en la experiencia de relación entre el cliente y el producto. En esta investigación se establecieron 11 dimensiones de calidad de las cuales conocimiento, producto y personalización representan a las percepciones y sugieren que el método proporciona resultados fáciles de interpretar en el sector bancario. Los resultados resaltan la importancia de la inversión en gestión de calidad en las relaciones con los clientes con el servicio y puede ayudar a mejorar el grado de lealtad (Aldlaigan \& Buttle, 2002). 
El estudio de Galloway y Blanchard incorporara una perspectiva de hardware y software en la medición del servicio desarrollando un modelo alternativo basado en tres dimensiones el proceso, el grado de calidad del servicio y hardware/software que representa el aspecto físico y la relación interpersonal. Los resultados presentan similitudes con los estudios de Mersha \& Adlakha (1994) en la exactitud del servicio, el servicio realizado correctamente a la primera vez y el conocimiento sobre el servicio y aspectos como la tangibilidad y la apariencia del servicio se consideraron como de menor importancia, destacando la investigación en el servicio bancario y confirmando la fuerte relación con la calidad percibida de Cronin, la importancia de algunos atributos como el conocimiento, la eficiencia, la velocidad de atención (Ribeiro, Machado \& Tinoco, 2010).

En cada uno de estos modelos se destaca las cinco dimensiones propuestas en el modelo de SERVQUAL: tangibilidad, garantía, capacidad de respuestas, seguridad y empatía incluyendo las variables Tecnología, Conectividad, Cobertura y Accesibilidad. La variable Tecnología es resaltada en la variable de los servicios de cajeros automáticos, la cual propone soluciones técnicas y sistematizadas en las máquinas como elementos de la calidad del servicio y cubre también a las nuevas variables (Berdugo, Barbosa \& Prada, 2016).

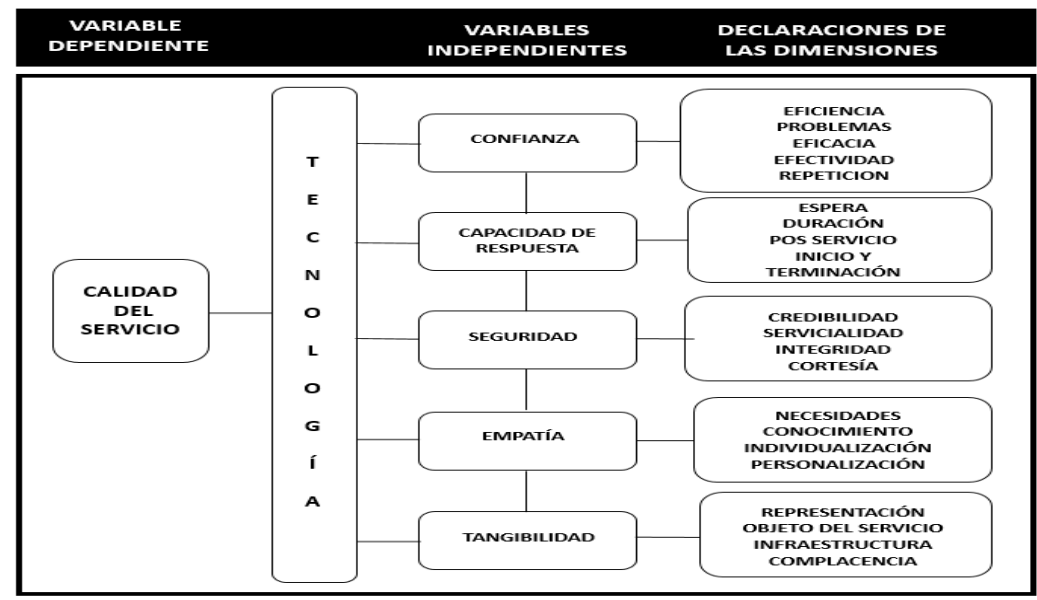

Gráfico 2. Dimensiones de la calidad bancaria

Elaborado por: autores

El Gráfico 2 presenta la relación del modelo de Cronin \& Taylor (1992) en conjunto con las aportaciones tecnológicas de los modelos de Avkiran (1994) y el modelo de Karatepe, Yavas y Babakus (2005) como un modelo válido para la medición de la calidad del servicio percibido usando la escala de SERVPERF de 22 ítems para representar las cinco dimensiones y elaborar que mide a cada uno de los indicadores.

\section{Materiales y métodos}

Para los autores la investigación analizó las características del fenómeno observado en cada uno de sus indicadores en el uso de los servicios de cajeros automáticos como un estudio descriptivo y correlacional a partir de un marco de trabajo que proporciona una fotografía de la situación problemática que fue de gran utilidad al 


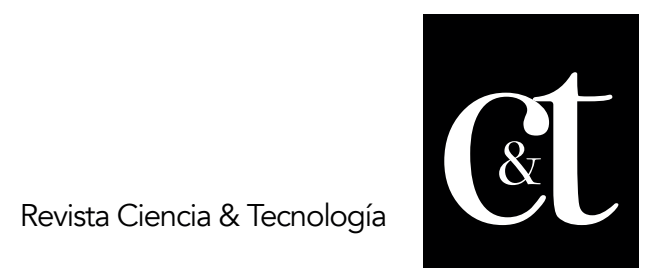

No. 28,31 de octubre de 2020

ISSN impreso: 1390 - 6321

ISSN online: 2661 - 6734

medir sus componentes y el grado de asociación que existe entre las dimensiones a partir del objeto de estudio en forma cualitativa y cuantitativa (Carrasco, 2013).

El enfoque fue cuantitativo en la investigación porque permitió medir con precisión sus datos y analizar, evaluar y recopilar los datos de las variables con métodos estadísticos, fue deductivo por la necesidad de descomponer el objeto de estudio en cada una de sus partes. Por su naturaleza cuantitativa la investigación planteó la comprobación de los enunciados siendo la construcción de hipótesis para explicar si el fenómeno observado fue válido o nulo. El tipo de investigación fue sistemática y empírica es decir no experimental con un corte longitudinal de tendencia en un periodo especificado que permitió realizar inferencias a los cambios (Valderrama, 2013).

\section{Variables latentes y observadas}

De manera conceptual lo que se intenta es plasmar un modelo que permita la identificación de factores en el desarrollo de nuevas tecnologías en la calidad del servicio de medios de pagos electrónicos tomando en consideración como estas variables son interrelacionadas en el entendimiento de la calidad del servicio que pudiera afectar al negocio, con lo cual los gerentes podrán apoyar al evaluar diversos planes de acción durante la fase de planeación de nuevos servicios en los cajeros automáticos. El uso de variables latentes que no son medidas directamente por un instrumento generalmente aceptado (Schumacker \& Lomax, 2004) contribuye a formar una variable denominada observadas o indicadores. Se asume que cualquier medición será imperfecta (Bollen, 1989) porque cada observación en el mundo real viene con un cierto error en la medición que se consideran como error aleatorio y error sistemático debido a la aplicación del método, por lo que el valor observado de un ítem es la suma de las tres partes llamadas, el verdadero valor de la variable, el error aleatorio y el error sistemático.

Identificación de las dimensiones de la calidad y construcción de la matriz de recolección de datos

En esta fase se identificó las dimensiones a partir de los estados de cada variable de la calidad percibida como se presenta en el Gráfico 2, manteniendo los 22 ítems del modelo SERVPERF para la variable confianza y tangibilidad.

Tabla 1. Dos estados de la percepción en la calidad percibida

\begin{tabular}{|c|c|c|c|c|c|}
\hline \multicolumn{2}{|c|}{ ESTADOS DE LA CONFIANZA } & \multirow{2}{*}{\begin{tabular}{|l|}
\multicolumn{1}{|c|}{ OBJETIVD DE LA } \\
DIMENSIÓN
\end{tabular}} & \multicolumn{2}{|c|}{ ESTADOS DE LA TANGIBILIDAD } & \multirow{2}{*}{\begin{tabular}{|l|}
\multicolumn{1}{|c|}{ OBJETIVO DE LA } \\
DIMENSIÓN \\
$\begin{array}{l}\text { Determinar el nivel de } \\
\text { pulcritud que se ofrece en } \\
\text { el servicio }\end{array}$ \\
\end{tabular}} \\
\hline Eficiencia & $\begin{array}{l}\text { Cuando en el servicio se } \\
\text { le promete algo en cierto } \\
\text { tiempo. }\end{array}$ & & Representación & $\begin{array}{l}\text { Las instalaciones físicas son } \\
\text { visualmente atractivas. }\end{array}$ & \\
\hline Problemas & $\begin{array}{l}\text { El Banco tiene interés en } \\
\text { solucionar un problema } \\
\text { del los clientes en el uso } \\
\text { del servicio. }\end{array}$ & $\begin{array}{l}\text { Agilidad en la solucion de } \\
\text { errores }\end{array}$ & Objeto & $\begin{array}{l}\text { El Producto Asociado con el } \\
\text { servicio es atractivo. }\end{array}$ & $\begin{array}{l}\text { Determinar el grado de } \\
\text { percepción visual de los } \\
\text { cajeros automáticos. }\end{array}$ \\
\hline Eficacia & \begin{tabular}{|l|}
$\begin{array}{l}\text { Se presta habitualmente } \\
\text { bien el servicio. }\end{array}$ \\
\end{tabular} & \begin{tabular}{|l}
$\begin{array}{l}\text { Cumplimiento de lo } \\
\text { acordado }\end{array}$ \\
\end{tabular} & Infraestructura & $\begin{array}{l}\text { El servicio se ofrece con } \\
\text { tecnologías de apariencia } \\
\text { moderna. }\end{array}$ & $\begin{array}{l}\text { Identificar los tipos de } \\
\text { tecnología bancaría } \\
\text { existente en los cajeros } \\
\text { automáticos. }\end{array}$ \\
\hline Efectividad & $\begin{array}{l}\text { EI servicio es el acordado } \\
\text { con el cliente. }\end{array}$ & $\begin{array}{l}\text { Expectativa del servicio } \\
\text { diseñado }\end{array}$ & \multirow{2}{*}{ Complacencia } & \multirow{2}{*}{$\begin{array}{l}\text { Los elementos, materiales y } \\
\text { documentación relacionada } \\
\text { con los servicios que ofrecen } \\
\text { son atractivos y sencillas. }\end{array}$} & \multirow{2}{*}{$\begin{array}{l}\text { Identificar el grado de } \\
\text { preferencias de los } \\
\text { documentos entregados } \\
\text { para los servicios. }\end{array}$} \\
\hline Repetición & $\begin{array}{l}\text { El servicio mantienen sus } \\
\text { registro sin errores. }\end{array}$ & $\begin{array}{l}\text { Funcionamiento del servicio } \\
\text { en la primera vez }\end{array}$ & & & \\
\hline
\end{tabular}

Elaborado por: autores 
A partir de cada uno de sus estados se construyó la matriz de datos cuya función principal fue guiar la operacionalización de cada una de sus variables desde un nivel abstracto a un plano concreto para ser medidas y analizadas, descomponiéndolas en dimensiones, indicadores, su forma de recolectar, archivo y procesamiento como se muestra en el cuadro No. 4 para lograr una investigación lo más "objetiva" posible.

\section{Tabla 2. Estructura de la matriz de recolección de datos}

\begin{tabular}{|l|l|}
\hline \multicolumn{2}{|c|}{ ESTRUCTURA DE LA MATRIZ OPERACIONALIZACIÓN } \\
\hline Variable dependiente & Variable del desenlace deinterés que se pretende explicar o estimar \\
\hline Variable independiente & Variable que se mide para determinar el valor de la variable dependiene \\
\hline Declaración de la variable & Definción conceptual \\
\hline Dimensión & Aspectos del problema a investigar \\
\hline Objetivo de la dimensión & Especifica que aspecto se va a investigar \\
\hline Declaración de la dimensión & Parámetros que contribuyen a ubicar la situación de la problemática planteada \\
\hline Indicador & Nombre del indicador \\
\hline Declaración del indicador & Cuantificación de la dimensión que es susceptible a adoptar valores o ser expresada en categorías \\
\hline Item & Es la pregunta de como se debe buscar la información del problema a investigar \\
\hline Frecuencia & Periodo de medición en años, meses días y horas \\
\hline Técnica & Tipo de recolección: Campo, estadístico o documental \\
\hline Instrumento & Encuestas, entrevistas, base estadística o lista de cotejo \\
\hline Fuente & Origen de la información: primaria o secundaria \\
\hline Tipo de variable & Variable cualitativa o cuantitava \\
\hline Tipo de escala & Nominal, ordinal, de intervalo o de razón \\
\hline Identificación del indicador & Nombre técnico asignado al indicador para el procesamiento de la información \\
\hline Descripción del identificador & Significado del nombre técnico para el procesamiento de la información \\
\hline Procesamiento del dato & Estadístico SPSS, consolidado del registro transaccional \\
\hline Fichero & Nombre del archivo electrónico que contiene la información del problema a investigar \\
\hline
\end{tabular}

Elaborado por: Autores

Las preguntas o ítems que permitieron medir cada una de las dimensiones fue ajustada mediante una prueba piloto aplicada con técnicas de campo y una validación de contenido.

\section{Recolección y tratamiento de datos}

La dimensión de la calidad se midió usando el enfoque de las percepciones y sus resultados se transformaron para su interpretación mediante la escala de Likert con una escala de 1 a 7 basado en la evidencia de nuevos estudios de Brady \& Cronin (2001) de que las medidas de solo percepciones de la calidad del servicio son superiores a otros enfoques.

Los datos fueron recolectados de las bases estadística de la Superintendencia de Bancos, el Banco Central del Ecuador durante un periodo de dos años analizando principalmente la información de cantidad de transacciones, clientes, tarjetas de débito, cajeros y su geo-localización y de manera focal se seleccionó uno de los bancos más crecientes en servicios de ATM basándose en el criterio de mayor cantidad de tarjetas de débito sin chip en la banca ${ }^{4}$, que permitió identificar los

\footnotetext{
${ }^{4}$ Superintendencia de Bancos del Ecuador. (2017). Estadísticas Generales Servicios Financieros - Tarjetas de débito. Recuperado de: http://oidprd.sbs.gob.ec/practg/sbs_index?vp_art_id=9914\&vp_tip=2\#ATM
} 


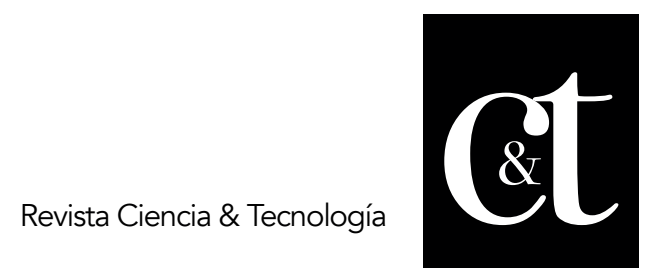

No. 28,31 de octubre de 2020

ISSN impreso: 1390 - 6321

ISSN online: 2661 - 6734

niveles de eficiencia, espera, duración, credibilidad integridad, necesidades del cliente e infraestructura.

Con el propósito de validar la asociación en las variables recopiladas con datos cualitativos se recurrió al coeficiente de contingencia $C$ de Karl Pearson cuya relación matemática se presenta en la ecuación 1 (Spiegel y Stephens, 2009) y representa la medida del grado de asociación o relación entre dos conjuntos o atributos con la misma escala nominal en la tabla de contingencia estableciendo el nivel de independencia o asociación entre ambas variables.

$$
C=\sqrt{\frac{\chi^{2}}{\chi^{2}+N}}
$$

Donde " $\mathrm{N}$ " es el tamaño de la muestra y el campo de variación del coeficiente " $\mathrm{C}$ " queda acotado entre cero y uno siendo la proximidad a cero del coeficiente como un menor grado de asociación y cero como un caso extremo de independencia.

Para procurar certeza y confiabilidad en el tratamiento de la información numérica se utilizó análisis estadísticos con la herramienta IBM SPSS para datos agrupados como tabla de distribución de frecuencias, tabla inferencia estadística con intervalo de confianza, aplicación de la prueba t de student, análisis de varianza y tablas de contingencia. En el caso de las muestras fue preciso calcular los niveles de significación de las diferencias que aparecen entre los porcentajes y los promedios, y en caso de aparecer diferencias fue necesario conocer los límites dentro de las cuales estas diferencias son estadísticamente significativas, como instrumento de medición de los datos finales fue necesario llevar los resultados a la escala de Likert para medir y registrar con precisión cada uno de los indicadores asociados a las propiedades o característica del fenómeno.

\section{Resultados}

Valores de variables y dimensiones identificadas en el estudio:

Tabla 3. Confianza de los servicios ofrecidos

\begin{tabular}{|l|c|r|}
\hline VARIABLE & DIMENSIÓN & \multicolumn{1}{|c|}{ RESULTADO } \\
\hline \multirow{3}{*}{ Confianza } & Eficiencia & $77,60 \%$ \\
\cline { 2 - 3 } & Problemas & $78,95 \%$ \\
\cline { 2 - 3 } & Eficacia & $98,81 \%$ \\
\cline { 2 - 3 } & Efectividad & $91,07 \%$ \\
\cline { 2 - 3 } & Repetición & $100,00 \%$ \\
\hline
\end{tabular}

Elaborado por: autores

En el análisis longitudinal de los datos se observó que la dimensión eficiencia es el indicador con menor resultados al medir que se cumpla con el servicio acertadamente, que sus tiempos de atención a los reclamos estén acordes a los niveles adecuados, que se cumpla con lo acordado por el cajero, se entregue el 
servicio acordado, que el cliente utilice frecuentemente el servicio de la tarjea de débito en otra identidad.

El hallazgo en la dimensión eficiencia (77.60\%) desde la percepción del cliente fue necesario contestar a la pregunta si "el cajero automático cumple el servicio acertadamente", lo que conllevó a identificar y medirla desde la disponibilidad del servicio en base a 5 estados de eficiencia (Tabla 4).

Tabla 4. Disponibilidad de los servicios ofrecidos

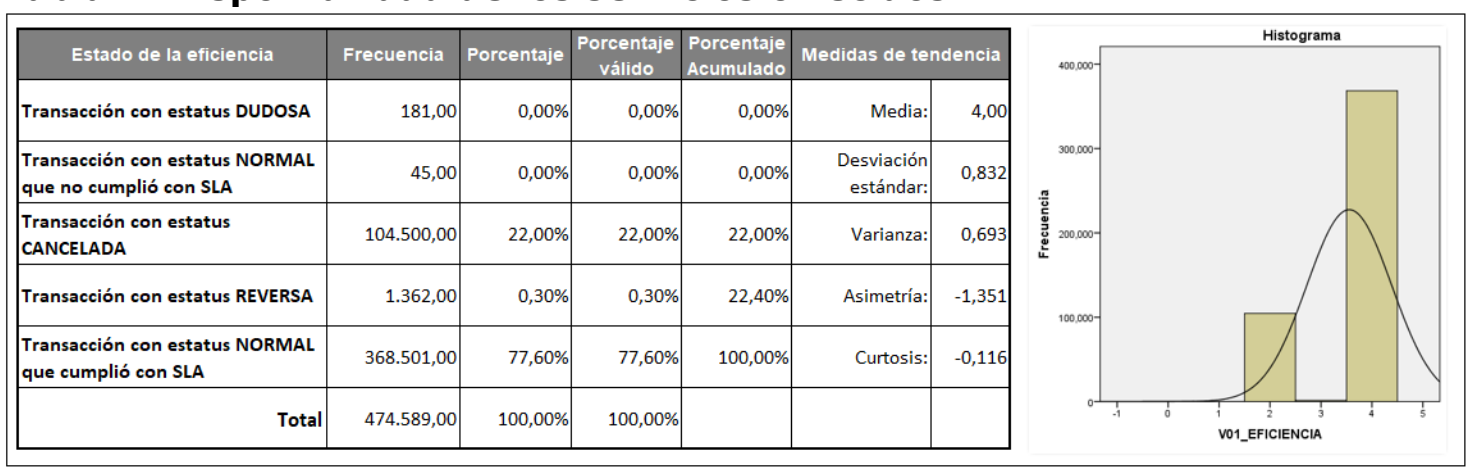

Elaborado por: autores

Tabla 5. Capacidad de respuesta

\begin{tabular}{|c|c|r|}
\hline VARIABLE & DIMENSIÓN & RESULTADO \\
\hline \multirow{3}{*}{ Velocidad de respuesta } & Espera & $84,00 \%$ \\
\cline { 2 - 3 } & Duración & $99,90 \%$ \\
\cline { 2 - 3 } & Pos Servicio & $17,60 \%$ \\
\cline { 2 - 3 } & Inicio y Terminación & $95,97 \%$ \\
\hline
\end{tabular}

Elaborado por: autores

Esta dimensión (Tabla 5) determinó si el banco se anticipa a los problemas que pueda presentar el cliente como el tiempo de espera y de repuesta al cliente al momento de utilizar el cajero automático, seguimiento de sus novedades reportadas y si el banco siempre está dispuesto a ayudar los clientes de los cajeros automáticos. Se identificó que la dimensión que requiere de atención es Pos-Servicio, qué se refiere a la proactividad en el servicio ofrecido, seguimiento a los eventos erróneos y anómalos.

Tabla 6 Seguridad del servicio

\begin{tabular}{|c|c|r|}
\hline VARIABLE & DIMENSIÓN & RESULTADO \\
\hline \multirow{3}{*}{ Seguridad } & Credibilidad & $77,60 \%$ \\
\cline { 2 - 3 } & Servicialidad & $77,80 \%$ \\
\cline { 2 - 3 } & Integridad & $77,70 \%$ \\
\cline { 2 - 3 } & Cortesía & $66,66 \%$ \\
\hline
\end{tabular}


Esta dimensión determina si el banco brinda certeza en las transacciones de los clientes en los cajeros automáticos, si los cajeros cuentan con información para que los clientes puedan atender sus necesidades ante un evento inusual que se representó con un $66.66 \%$ en su resultado siendo la forma de comunicar los reclamos originados en los canales electrónicos.

\section{Tabla 7. La empatía como el conocimiento de las necesidades que proporciona el servicio a sus clientes}

\begin{tabular}{|l|c|r|}
\hline VARIABLE & DIMENSIÓN & \multicolumn{1}{|c|}{ RESULTADO } \\
\hline \multirow{4}{*}{ Empatía } & Necesidades del cliente & $36,60 \%$ \\
\cline { 2 - 3 } & Conocimiento del cliente & $46,00 \%$ \\
\cline { 2 - 3 } & Individualización & $29,14 \%$ \\
\cline { 2 - 3 } & Personalización & $100,00 \%$ \\
\cline { 2 - 3 } & Horario Flexibles & $76,43 \%$ \\
\cline { 2 - 3 } & &
\end{tabular}

Elaborado por: autores

El análisis de esta dimensión evaluó las opciones que utilizan los clientes en el servicio, sus nuevas necesidades, el grado de individualización que le brinda el servicio representando por un menú u opciones personalizadas e identificar si el servicio ofrecido está disponible en horarios no convencionales.

Tabla 8. Nivel de complacencia de los elementos tangibles

\begin{tabular}{|c|c|r|}
\hline VARIABLE & DIMENSIÓN & \multicolumn{1}{c|}{ RESULTADO } \\
\hline \multirow{2}{*}{ Tangibilidad } & Representación del servicio & $76,60 \%$ \\
\cline { 2 - 3 } & Objeto del servicio & $98,80 \%$ \\
\cline { 2 - 3 } & Infraestructura & $62,50 \%$ \\
\cline { 2 - 3 } & Complacencia & $33,31 \%$ \\
\hline
\end{tabular}

Elaborado por: autores

Esta variable representó en el estudio el nivel de pulcritud que se ofrece en el cajero automático, que el producto asociado sea atractivo, que sus tecnología sea de apariencia moderna y finalmente que sus elementos y materiales identifiquen el grado de aceptación y nivel de complacencia del servicio que vio reflejando en la aceptación de los modelos de tarjetas electrónicas, claridad y entendimiento en los recibos entregados y finalmente la calidad de los billetes ofrecidos en la transacción.

\section{Discusión de los resultados}

En el estudio de la percepción de la calidad del servicio en cajeros automáticos se aplicó el modelo de Cronin y Taylor con las aportaciones de los modelos de Avkiran; 


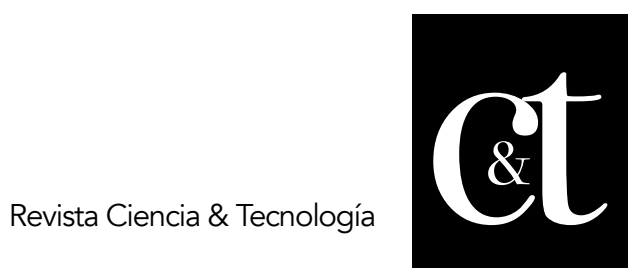

No. 28,31 de octubre de 2020

ISSN impreso: 1390 - 6321

ISSN online: 2661 - 6734

Karatepe, Yavas y Babakus, el cual permitió identificar factores críticos de la calidad del servicio en el uso de los cajeros automáticos de las instituciones bancarias.

En la investigación se consideró las 22 variables del modelo propuesto analizándolas en función de las transacciones de los cajeros automáticos del sistema bancario mediante pruebas estadísticas, análisis matemáticos y cálculos porcentuales determinando una escala de puntaje por cada dimensión, lo que permitió identificar que dimensiones se consideran como un factor crítico en el servicio de cajeros automáticos para ser mejorados con estrategias operacionales y tecnológicas.

Debido a que las instituciones financieras no se anticipan a los reclamos que originan las transacciones fallidas el cual se evidenció en el análisis efectuado con la variable velocidad o capacidad de respuesta de la dimensión Pos servicio cuyo valor de 2 puntos que representa una ponderación del $17.60 \%$ como se muestra en el cuadro No 7 y que ocupa la escala más baja en relación a las 21 variables.

En la valoración de la variable empatía se encontró tres dimensiones con puntaje bajos acorde a la escala aplicada, las mismas que son: necesidades del cliente, conocimiento e individualización.

Para la valoración de las relaciones que tiene las instituciones financieras con sus clientes se consideró la dimensión Individualización tomando como dato para la investigación la cantidad de clientes y la cantidad de tweets o mensajes de los usuarios siendo esta inferior por lo alcanzó un valor de $29.14 \%$, que representó 3 puntos en la escala de Likert.

Las transacciones de retiros, consultas y transferencias son las más utilizadas en los cajeros automáticos, sin embargo, existen transacciones que no son utilizadas como los pagos de servicios y compras de tarjetas prepagos por lo expuesto la dimensión Necesidad del cliente permitió reconocer que servicios no son utilizados en los cajeros automáticos y obtuvo un valor cuantitativo de $36 \%$ en la escala de Likert.

La importancia en búsqueda de nuevos servicios en función de los intereses de los clientes se pudo medir con la dimensión Conocimiento del cliente el cual obtuvo una ponderación del $46 \%$ debido a que las instituciones financieras realizan sus implementaciones basándose solo con el comportamiento dentro de la institución del cliente.

Finalmente, la variable tangibilidad en la valoración de la dimensión Complacencia la cual se encuentra relacionada con los instrumentos utilizados en las transacciones de los cajeros automáticos representó en su evaluación el valor de $33.31 \%$, debido a que se consideró como los instrumentos necesarios el billete el cual es un objeto primordial en la transacción de retiro y la impresión del recibo cuando el cliente lo solicita. 


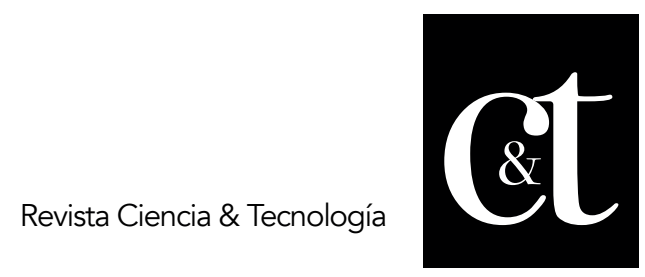

No. 28,31 de octubre de 2020

ISSN impreso: 1390 - 6321

ISSN online: 2661 - 6734

Una vez identificados los factores críticos de la calidad del servicio de cajeros automáticos se identificó mejoras basándose en las variables con valores menores a $50 \%$.

Gestión de clientes, mejorará la dimensión conocimiento al cliente y la individualización debido a que, con el análisis de la información no estructurada, información que se encuentra en la Web permitirá la incorporación de nuevos servicios que serán diseñados de acuerdo a la necesidad del cliente analizado desde un contexto de conocimiento y comportamiento general.

Gestión de transacciones erróneas, permitirá mejorar la dimensión Pos-servicio al incorporar la información interna a la tecnología Big data permitirá obtener de manera oportuna los reclamos de los clientes y gestión interna para procesos de devolución del dinero en casos necesarios.

Los mantenimientos y la dotación de los materiales necesarios forman parte de la cadena de valor del servicio de cajeros automáticos y que fueron medidos con la dimensión complacencia, siendo este un indicador critico en la calidad del servicio se considera la incorporación de tecnología Big data para mejorarlo ya que con el uso de tecnología analítica y predictiva se podrá identificar el tiempo, periodicidad apropiada para efectuar estos mantenimientos.

También se determinó la correlación de sus variables para establecer la correspondencia de los datos recopilados considerando para este análisis las dimensiones Eficiencia y Duración con pruebas estadísticas de chi cuadrado, coeficiente de contingencia, correlación de Mathew y coeficiente de Cramer en el que se midió su grado de asociación lineal. Se estableció 3 hipótesis para comprobar la evidencia muestral que fueron orientadas a las variables Eficiencia, Empatía y Duración del servicio las mismas que en unos casos se rechazó la hipótesis nula y en otros se comprobó hipótesis nula.

\section{Conclusiones}

En base a los factores identificados en el servicio de cajeros automáticos, una la propuesta se enfoca en tres aristas globales en el servicio de cajeros automáticos que contiene las siguientes variables:

Asegurar la cadena de valor en las transacciones de cajeros automáticos considerando importante los mantenimientos y abastecimiento de suministro de los cajeros en relación a mejorar la variable tangibilidad en la dimensión complacencia.

Gestionar las transacciones erróneas de tal manera que se pueda anticipar los reclamos de los clientes, mediante la variable velocidad o capacidad de respuesta en su dimensión pos-servicio. 


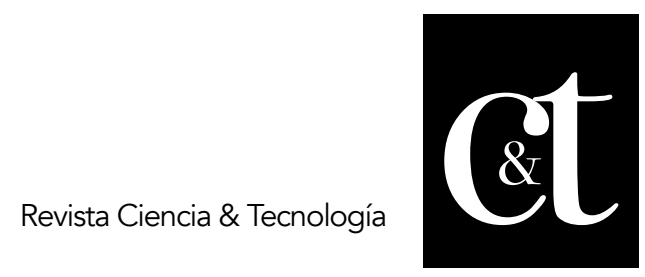

No. 28, 31 de octubre de 2020

ISSN impreso: 1390 - 6321

ISSN online: 2661 - 6734

Identificar las necesidades de los clientes en cajeros automáticos mediante la mejora de la variable empatía en sus dimensiones nuevas necesidades, conocimientos e individualización de los mismos.

\section{Referencias bibliográficas}

Aldlaigan, A.H. \& Buttle, F.A. (2002). SYSTRA-SQ: a new measure of bank service quality. International Journal of Service Industry Management, Vol. 13 No. 4, pp. 362-81.

Avkiran, N. K. (1994). Developing an Instrument to Measure Customer Service Quality in Branch Banking. International Journal of Bank Marketing, 12:10-18.

Berdugo, C., Barbosa, R. y Prada, L. (2016). Variables relevantes para la medición de la calidad percibida del servicio bancario. Disponible en https://search.proquest.com/docview/1802641358?accountid=130066 [consulta: 5-11-2018]

Bollen, K. (1989). Structural Equations with Latent Variables. John Wiley \& Sons, Inc.

Brady, M. \& Cronin, J. J. (2001). Some new thoughts on conceptualizing perceived services quality: a hierarchical approach. Journal of Marketing, 65, 34-49.

Carrasco, S. (2013). Metodología de la investigación científica. Tercera Edición. Lima. Editorial Fondo UNMSM.

Cronin, J.J. \& Taylor, S.A. (1992). Measuring Service Quality: A Reexamination and Extension. Journal of Marketing, 56, pp. 55-68.

Del Molino, P. (2010). Modelo Q+4D: Como medir la satisfacción del cliente más allá de la calidad percibida. Madrid: España. Editorial Aenor. Asociación Española de Normalización y Certificación.

Ennew, C. \& Binks, M. (1996). The Impact of Service Quality and Service Characteristics on Customer Retention: Small Businesses and Their Banks in the UK. British Journal of Management, 7(3):219 - 230.

Grande, I. (2005). Marketing Estratégico. Cuarta Edición. Madrid: España. Editorial ESIC.

Grönroos, C. (1984). A Service Quality Model and its Marketing Implications. European Journal of Marketing, 18(4), 36-44.

Kano, N. (1984). Attractive quality and must-be quality. The Journal of the Japanese Society for Quality Control, 14.2 (1984), págs. 39-48

Karatepe, O.M., Yavas, U., \& Babakus, E. (2005). Measuring service quality of banks: Scale development and validation. Journal of Retailing and Consumer Services, Vol. 12 , pp. 373-383.

Mersha, T., \& Adlakha, V. (1992): Attributes of service quality: the consumers' 


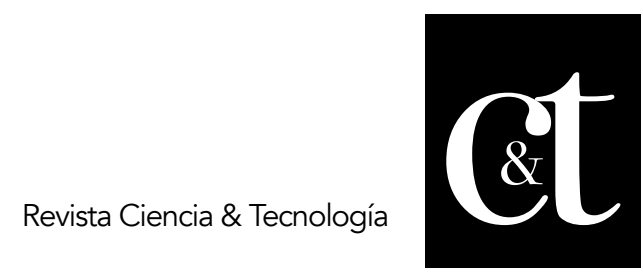

No. 28, 31 de octubre de 2020

ISSN impreso: 1390 - 6321

ISSN online: 2661 - 6734

perspective. International Journal of Service Industry Management, vol. 3, n० 3, pp. $34-45$.

Parasuraman, A., Zeithaml, V., \& Berry, L. (1988). SERVQUAL: a multiple-item scale for measuring consumer perceptions of services quality. Journal of Retailing, 64(1), pp. 12-40.

Ribeiro, José Luis Duarte; Machado, Cássio Oliveira; \& Tinoco, Maria Auxiliadora Cannarozzo. (2010). Satisfaction determinants and quality attributes in bank services. Gest. Prod. [online]. Vol.17, n.4, pp.775-790.

Sangeetha, J. \& Mahalingam, S. (2011), Service quality models in banking: a review, International Journal of Islamic and Middle Eastern Finance and Management, Vol. 4 Iss: 1 pp. $83-103$

Schumacker, R. \& Lomax, R. (2004). A beginner's guide to structural equation modeling (2nd ed.). Lawrence Erlbaum Associates.

Spiegel, M. y Stephens, L. (2009). Estadística. 4ta. Ed. México: Mc Graw Hill.

Valderrama, S. (2013). Pasos para elaborar proyectos de investigación científica. Cuantitativa, Cualitativa y Mixta. Segunda edición. Lima. Editorial San Marcos. 